\title{
Labyrinthe
}

9| 2001

Numéro 9

\section{Le café parisien}

Échanges de vues

Joanne Vajda

\section{(2) OpenEdition}

Journals

Édition électronique

URL : http://journals.openedition.org/labyrinthe/931

DOI : 10.4000/labyrinthe.931

ISSN : 1950-6031

Éditeur

Hermann

Édition imprimée

Date de publication : 30 juin 2001

Pagination : 59-76

Référence électronique

Joanne Vajda, «Le café parisien », Labyrinthe [En ligne], 9 | 2001, mis en ligne le 30 janvier 2006

consulté le 10 décembre 2020. URL : http://journals.openedition.org/labyrinthe/931 ; DOI : https:// doi.org/10.4000/labyrinthe.931

Ce document a été généré automatiquement le 10 décembre 2020.

Propriété intellectuelle 


\title{
Le café parisien
}

\author{
Échanges de vues
}

Joanne Vajda

1 Le café n'est en réalité qu'un prétexte pour s'interroger sur les relations qu'un tel lieu entretient, à travers son architecture, avec la ville et la société. L'entre-deux-guerres est pour les cafés parisiens une période faste, paradoxalement peu étudiée. Aux côtés de caractéristiques constantes, acquises par ces établissements dès la fin $d u \mathrm{XVIII}^{\mathrm{e}}$ siècle, apparaissent des éléments nouveaux, propres à la période 1920-1940.

2 La source privilégiée pour nous éclairer sur l'espace des cafés est la littérature. Mais les publicités, les textes de guides ou les articles de presse ont leur place parmi les témoins. Impossible aussi de se dispenser de l'image. L'analyse s'appuie sur des documents visuels : photos, dessins, peintures et autres pièces graphiques ${ }^{1}$.

3 L'histoire des cafés s'écrit habituellement dans trois registres voisins et solidaires, le registre de l'histoire sociale, le registre de l'histoire culturelle et celui de l'histoire des subjectivités. Les cafés représentent « des académies, des vitrines, des Larousse [...], et comme une table des matières de l'histoire d'une ville et d'une époque $»^{2}$. Il y a, parallèlement, une histoire de l'architecture des cafés, racontée au moyen de concepts théoriques et de principes architecturaux. À travers cette analyse nous avons saisi les interférences entre toutes ces histoires. La 4 e dimension du café, l'architecture, prend ici toute son importance. La perte d'identité actuelle de l'espace du café accentue l'idée qu'il s'agit d'un lieu « familièrement inconnu ». Pourtant, pendant longtemps, sa décoration a contribué à la définition de sa personnalité, autant que les personnages qui le fréquentent. C'est pourquoi nous examinons les rapports que le café cultive avec la clientèle et avec les espaces avoisinants - le salon et la rue. Le constat de la banalisation rend d'autant plus importante l'appréhension des relations entre le café parisien et l'air du temps. Son architecture s'accorde avec l'esprit de l'époque.

4 L'approche envisagée est thématique, ce qui permet de voir comment architecture et société s'édifient l'une par rapport à l'autre. La recherche historique prend ainsi une forme accessible au profane et rappelle les conversations entendues au café.

Regards sur l'histoire 
5 Les appellations sous lesquelles on retrouve les débits de boissons urbains sont nombreuses ${ }^{3}$. À partir de la seconde moitié du xix ${ }^{e}$ siècle, le mot café devient le plus usuel ${ }^{4}$

6 La particularité des espaces qui se développent entre 1920 et 1940 est qu'ils regroupent plusieurs fonctions. On y consomme des boissons en tous genres, on y mange, on s'amuse et on y danse même. La modernité se manifeste dans ces nouveaux programmes : des constructions audacieuses apparaissent, comprenant à la fois un bar, un café, une brasserie, un restaurant et un dancing. Les ensembles, se développant souvent sur plusieurs niveaux, se nomment café-restaurant, bar-brasserie, restaurant-dancing, etc.

7 Il convient de préciser que notre regard s'est principalement arrêté sur les espaces qui présentent un certain intérêt architectural. L'architecte Robert Mallet-Stevens, résume ce point de vue : il y a " d'abord une salle quelconque dans laquelle des gens mangent. Ensuite une pièce camouflée en " vieille maison », en « hostellerie » où le client déguste, dans un cadre correspondant à l'origine, des plats plus ou moins savamment préparés. Enfin, une architecture bien éclairée, bien ventilée dans laquelle le consommateur repose ses yeux sur un spectacle dont les principaux acteurs sont les autres consommateurs ». Il ajoute : « il n'y a rien à dire sur la première catégorie puisqu'elle est nulle et ne présente aucun intérêt. Quant à la deuxième, l'hostellerie, elle a de plus en plus la vogue et représente hélas l'art décadent qui sévit un peu présentement [...]. Le cadre est presque toujours ridicule. Le régionalisme alimentaire a créé une espèce de régionalisme en architecture [...]. Il y a heureusement, à côté de ces restaurants de kermesse, des restaurants étudiés, d'une architecture normale $»^{5}$.

8 Après la Première Guerre mondiale, une nouvelle société apparaît, plus superficielle, aimant le luxe, l'excès et le tumulte. Elle naît d'un « brassage sans précédent de peuples et de classes ${ }^{6}$. Les Champs-Élysées deviennent la vitrine de l'espace cosmopolite de l'entredeux-guerres. La crise de 1929 porte un coup dur aux commerces, car nombreux sont les étrangers obligés de retourner dans leur pays. Malgré cela, un certain enthousiasme persiste et les cafés continuent à fleurir. La période qui suit est féconde pour quelques artistes et architectes qui luttent contre l'enfermement et l'académisme. Ceux-ci emploient un vocabulaire stylistique qui intègre, en l'interprétant, le registre architectural classique.

9 À partir de 1935, une vague de nationalisme envahit le pays. Les cafés gardent leur caractère égalitaire et cosmopolite, mais la verve qui y régnait disparaît peu à peu.

Face à face salon-café

10 Le café commence à faire concurrence à un autre lieu d'échanges et de discussions, le salon. Les changements de mentalités et le désir d'affirmation de la personnalité font que ce dernier tombe en désuétude, devenant « l'école du poncif et de la mode imbécile », à la différence du café, « l'école de la franchise et de la drôlerie spontanée ${ }^{7}$. Le salon perd son pouvoir ; désormais, c'est au café que les gloires se font et se défont. L'atmosphère du salon devient monotone et conventionnelle, alors que celle du café est vivante, nonconformiste et pleine de saveur. Au café on peut être soi-même, au contraire du salon où une certaine hiérarchie sociale règne et impose la conduite. Cependant les deux types d'espace, le salon et le café, coexistent. L'accès aux salons n'est pas permis à tout le monde, tandis que le café devient un lieu de rencontre ouvert au tout venant, l'une des plus importantes institutions parisiennes. Au Sélect, sur les Champs-Élysées, « on parlait 
millions [...]. Et pourtant, il était plus que certain que le plus important de ces personnages n'avait ni bureau, ni employés, ni domicile $»^{8}$.

11 Le café constitue l'annexe d'innombrables demeures car on peut y vivre sans contraintes. La boisson amplifie l'espace et ouvre les portes de l'imaginaire, offrant au consommateur une évasion immobile. L'influence des cafés sur la littérature passe par la conversation. Sa puissance sur l'art de même. Mais la plupart des discussions que l'on a au café, souvent très intéressantes et pleines d'idées, se perdent. " Pour remédier à cette frivolité, le George- $V$ lancera en 1938 le repas-disque ! À la demande des clients toutes les conversations seront enregistrées entre les hors-d'œuvre et le café. ${ }^{9}$ "

Ambiances et mentalités. Aperçu

12 Les guides touristiques de l'époque insistent de plus en plus sur la qualité du décor et sur le genre de public qui fréquente les cafés. Ils procurent des indications précises : Lipp «Brasserie obscure, mais fort achalandée. Beaucoup d'hommes de lettres, d'éditeurs, d'étudiants, de libraires et d'acteurs. C'est ici un royaume de littérature $»^{10}$.

13 Les cafés à la mode sont fréquentés par une population hétéroclite : habitués, mais aussi mannequins, gens de théâtre, magnats du cinéma, hommes d'affaires, dandys ou membres de la haute société. Les étrangers sont nombreux, qu'ils soient «naturalisés parisiens » ou « touristes ». Le café devient, avec l'hôtel, l'espace le plus cosmopolite de la ville.

14 Les architectes deviennent plus inventifs et plus soucieux de trouver des formes originales d'expression. Paradoxalement, des ensembles qui auraient paru ahurissants aux générations précédentes renouent avec la tradition parisienne des cafés exquis et charmeurs du XIX ${ }^{e}$ siècle $^{11}$. L'imagination et l'ingéniosité des concepteurs sont reconnues et appréciées par la presse de l'époque. Cependant, de nombreux écrivains refusent de voir dans ces changements les gages d'un renouveau.

Dès la fin du XIX ${ }^{e}$ siècle l'apparition des femmes et des boissons américaines dans le café déroute les habitués et fait subir au décor de grandes transformations. On commence à s'entasser dans les cafés : « sur la piste trop étroite, des couples remuent en cadence [...]. Une foule nombreuse, incohérente, s'essaie à danser, sans grand succès, mais non sans plaisir. [...] Les garçons, armés de plateaux, naviguent avec peine $»^{12}$. En regardant à l'intérieur d'un café, l'impression est de contempler le monde à travers un kaléidoscope. On triche avec l'espace qui se multiplie à l'infini. L'un des plus grands spécialistes dans le domaine est Charles Siclis, cet architecte qui sait jouer à merveille avec la lumière, la surface, la couleur et le volume, afin de créer la meilleure ambiance et les plus beaux effets.

Regards sur la décoration

16 L'architecture des cafés est une architecture éphémère, vouée aux transformations exigées par le goût du jour, lui-même en perpétuel changement. La pérennité ne satisfait plus et on ne s'attache plus au passé. Les entrepreneurs, comme dans d'autres domaines, ont fait des ravages : «Il regardait le Monoprix de la place Blanche. Ils l'ont agrandi en rien de temps. Y'a seulement six mois, on voyait le Paradis et l'Enfer, des cabarets ! [...] C'était beau ! Les Monuments Historiques auraient dû les classer. Le style 1900, pourquoi pas ? Un jour, y s'apercevront que c'était tonitruant et ils n'auront plus que des photos !» 13

17 Le café est un théâtre, un lieu de spectacle où le décor est aussi important que les acteurs. Les " méga-cafés ", voués au luxe et à la rencontre, éclosent dans toute la ville. 
L'automobile, l'aéroplane, le paquebot et le train, incarnations de la vitesse, deviennent des références pour la décoration des cafés. Les volumes étriqués se transforment en vaisseaux comme au Parnasse (Jean Michel, 1930) ou au Brébant (Arrighi, 1932, 32, boulevard Poissonnière), d'autres prennent des noms évocateurs, comme l'Aéroport (Blech, 1932), dancing en vogue à Montparnasse. Le dossier des banquettes de la brasserie Radio (Maurice Jallot, 1928, boulevard Clichy et rue Coustou) esquisse une succession de cellules distinctes qui rappellent les compartiments d'un wagon. Le mouvement est sans cesse exprimé, comme pour accentuer l'idée que le café est un lieu de passage.

Du rationalisme strict et rigide de la fin des années 1920, l'architecture des cafés évolue vers une simplicité moins rude jusqu'au milieu des années 1930. Celle-ci vire vers la douceur des courbes et la souplesse de l'ornement à la veille de la Deuxième Guerre. Les établissements qui voient le jour ont des allures très diverses, pleines d'esprit. L'intérêt pour la décoration des cafés augmente au point que l'on présente des "prototypes » à divers expositions et salons.

Le souci " hygiéniste " mène à l'emploi de matériaux qui donnent une impression de propreté, tels le verre, le métal, la céramique. Chaque intérieur de café brille grâce aux miroirs et aux métaux luisants, ce qui inspire des descriptions littéraires où les comparaisons les plus étonnantes sont faites, comme cette ébauche du Bouf sur le Toit (rue de Penthièvre) : "Mais dès le vestiaire on entre dans une ère d'éblouissements. D'immenses glaces superposées sur des plans différents jouent aux quatre coins de la salle principale. Grâce à une ingénieuse combinaison, elles sont encadrées de lumières rouges, bleues, vertes, violettes [...]. On se croirait dans la salle des Mirages au Musée Grévin ${ }^{14}$.

Entre 1920 et 1940 chaque idée est poussée à l'extrême. Par exemple, à l'opposé du café où l'on va pour passer du temps, on crée des établissements qui s'adressent à des consommateurs pressés. Le décor est soigneusement étudié afin qu'il ait l'air de découler des nécessités constructives, tel au bar Presto, situé sur les Grands Boulevards : « Mais le décor ? Eh bien, il n'y a pas de décor. [...] Ces gros tubes chromés qui encerclent le comptoir ? Un radiateur. La forme des guéridons, tels d'énormes champignons issus d'un massif cylindre ? Voulue, raisonnée : le cylindre sert de coffre pour recevoir les papiers usés. [...] et si la tablette du guéridon est épaisse, c'est pour contenir des lampes, car elle joint à son rôle celui de support des fonctions publicitaires et présente des annonces lumineuses. Oui, mais cet aquarium, une pure fantaisie ? Non pas [...] il amuse les passants et les incite à entrer $»^{15}$.

21 Aucun détail n'échappe au concepteur : l'entrée, la circulation de la clientèle et du personnel, la diffusion de la lumière, la disposition des tables, la scénographie qui organise le rapport intérieur-extérieur, la décoration, ainsi que les diverses questions techniques telles que chauffage, ventilation, aménagements de cuisines, etc. On adopte les " matériaux d'illusion, les miroirs qui multiplient les proportions, les lumières qui exaltent l'espace, [...] des cascades d'escalier, des ornements qui font chanter les murs ${ }^{16}$. Pour ce qui est de la décoration, « tout est sujet à étude : la mosaïque, les corniches, la lumière, les galeries porte-chapeaux, les chaises, les tables, les meubles, les lambrequins, les assiettes, les menus même! Quel champ immense pour le décorateur $!{ }^{17}$ Les piliers sont habillés de matériaux qui, par leur texture ou leur composition, les amenuisent en allant jusqu'à les dématérialiser.

La signalétique devient l'une des principales composantes de la façade, surtout grâce aux progrès faits à partir de 1914 dans l'élaboration des caractères de typographie. Le succès d'un tel établissement est assuré à partir du moment où le client, dès l'entrée, peut 
choisir sa place et voir tous les autres consommateurs, d'où l'importance accordée à l'étude des accès.

La circulation verticale est mise en exergue dans l'agencement des cafés. Ceux-ci se développent sur plusieurs niveaux, provoquant un va-et-vient continu des consommateurs qui contribue à l'impression générale d'agitation.

Les découvertes dans les domaines de la physique et de la médecine font que l'on se préoccupe davantage du cadre de vie. Dans une interview que Charles Siclis accorde à la revue Art et Médecine, celui-ci souligne que les essences agiraient différemment sur le système nerveux : la ronce d'acajou « excite la circulation du sang, le chêne stimule et le noyer déprime » et les bois exotiques grisent. Il explique aussi que la lumière exerce une action puissante sur le corps, de même que les couleurs. Aussi, " un grand volume vide « décomprime » l'individu surmené et qui aspire au repos $»^{18}$.

L'illumination des salles de café tient compte des dernières découvertes en la matière. L'éclairage indirect provient soit des corniches ou des fentes percées au plafond, soit des entailles pratiquées dans les murs, soit des verrières. Lorsqu'il est direct, il émane des lampes et appliques ou de l'extérieur, par l'intermédiaire des façades vitrées. Les tubes luminescents sont dissimulés dans les « plis » du plafond et produisent une ambiance intense et colorée.

La grande trouvaille des concepteurs consiste à utiliser les glaces comme matériaux d'architecture. L'espace s'accroît et l'éclairage s'accentue grâce à l'impression de multiplication des sources de lumière. «Effet de glaces, jeux de lignes, jeux de lumière, fausses perspectives à l'italienne, trompe l'œil ; c'est truqué comme un décor de féerie ${ }^{19}$. "

Les volumes des cafés, mettent en scène le spectacle de la ville. La salle prend la forme d'un amphithéâtre. L'architecte doit imaginer une atmosphère, des jeux de volumes et de matériaux pour le jeu des acteurs. Les regards des consommateurs se croisent dans les miroirs du café. L'espace devient irréel. Dans Le Colisée, café-restaurant sur les ChampsÉlysées (Charles Siclis, 1932), un subterfuge de perspective est employé, afin que le client n'ait pas l'impression de descendre en avançant vers le fond : le plancher monte, le plafond descend. Au Triomphe (Charles Siclis, 1934), la salle, divisée en gradins successifs, permet à tous les clients de voir et d'être vus, et de savourer le spectacle de l'avenue des Champs-Élysées.

Le savoir-faire progresse avec les connaissances techniques et scientifiques et avec l'industrialisation accélérée que connait le monde après la Première Guerre. Beaucoup de nouveaux matériaux sont employés : aluminium maté, métal Helvar, contre-plaqués de bois précieux, duralumin, fibracier, etc. Le « béton translucide » et les briques Nevada gagnent enfin une place méritée. La plupart des cafés qui voient le jour sont des " monuments » à la gloire du verre. Celui-ci apparaît sous tant de formes (miroir, vitrail, marbrite, glace opaque, transparente ou translucide, travaillée par différentes méthodes, gravure, sablage, etc.) qu'on n'a pas l'impression d'en abuser.

Les prouesses techniques se mettent au service de cette architecture : pour La Maxéville (1934), par exemple, on réalise le plus grand panneau de glace de planimétrie du monde, pour le Relais-Plaza (avenue Montaigne et rue du Boccador, 1936, Constant Lefranc, architecte) on emploie pour la première fois la glace aurée brute - glace à surface rugueuse d'aspect rosé - comme vitrage et revêtement extérieur.

Des tables de bakélite et d'acier chromé remplacent celles en bois et marbre. Les meubles de service sont en merisier jaune paille, en bois du Canada, ou en bois noir et sycomore 
blanc. Les sièges sont en métal blanc et recouverts de cuir, de velours rouge ou de tissu de crin vert.

D'autres modifications interviennent, notamment en ce qui concerne le comptoir, devenu bar. Il fait l'objet d'une industrie spéciale, étant traité de manière à ce que son image rappelle le moins possible celle du comptoir de bistrot en étain. Il se trouve habituellement à côté de l'entrée et occupe une grande partie de la salle. Les percolateurs et les pompes à bière métalliques meublent le bar et participent à l'ambiance générale.

La « fontaine lumineuse » est une invention de l'époque. Elle se dresse au centre de la salle, comme au Chiquito (Charles Siclis, 1928, rue du Colisée) ou Aux Armes de la ville (Rondoni, 1933, place de l'Hôtel de Ville). Avec le perpétuel mouvement et le jeu d'ampoules qui l'orne, elle participe à la création d'une ambiance gaie et optimiste autant que l'animation des murs et des plafonds. L'eau jaillit entre des dalles polies, éclairées à l'aide de projecteurs et de rampes électriques, en répandant l'éclat de l'ensemble dans la salle.

Café avec vue sur rue

L'apport du café à la voie publique est incontestable. Les toiles de Bonnard en témoignent : dans La Place Clichy (1912) et Le Café du Petit Poucet (1928), l'espace intérieur du café s'annexe la rue. Par l'architecture du café, le dehors s'engouffre à l'intérieur. Parallèlement, les devantures des cafés animent la ville, la dotant d'une âme et d'une silhouette.

Dès la fin du XIX $x^{e}$ siècle le plafond du café envahit la rue, se transformant en marquise métallique qui se continue par un auvent de toile, pour abriter la terrasse. Celle-ci constitue le prolongement de deux espaces qui se ressemblent tout en étant très différents : celui de la rue, des passants, et celui du café, des consommateurs. Le principe des gradins utilisé à l'intérieur des cafés de l'entre-deux-guerres s'applique également aux terrasses, ce qui vient conforter l'idée que le spectacle de la rue intéresse particulièrement les clients.

On essaie par tous les artifices de faire fondre les différences entre extérieur et intérieur. Une des plus remarquables devantures des années 1930 - à la fois inexistante et très présente - est celle de La Maison du Café, place de l'Opéra, conçue par Charles Siclis en collaboration avec le peintre Zarraga et le sculpteur Drivier. Elle sert de cadre à la décoration intérieure qui est une fresque. « Disposée, non à plat sur le mur du fond, mais selon une double sinuosité, en forme d's, elle parait de l'extérieur faire partie de la façade. C'est surtout du trottoir opposé qu'il faut la regarder - tout en marchant. Car, à mesure qu'il se déplace, le passant voit le triple cadre des baies vitrées glisser le long de l'œuvre peinte, et la composition générale ainsi découpée former les variantes successives de tableaux différents ${ }^{20}$. " La façade devient un élément dynamique de la composition. Elle n'est plus une limite et ne sert plus à circonscrire un espace, elle n'est plus qu'une protection contre les intempéries.

Des artifices de décoration font pénétrer l'extérieur jusque dans les salles de café situées au sous-sol, créant ainsi une situation paradoxale. Celles-ci sont souvent égayées par des dioramas représentant le ciel ou la mer. Au café Dupont du boulevard Barbès (Charles Siclis, 1935) la salle du sous-sol se nomme Belvédère car des dioramas représentant des images de Paris constituent son décor. « Sur les tables des consommateurs, des serviettes sur lesquelles sont imprimées des sortes de tables d'orientation donneront l'amusement 
au client de chercher l'emplacement des monuments. Ces dioramas peuvent être changés ${ }^{21} . »$

Tout ce monde mirifique et tout ce décor ahurissant se volatilisent à la fin d'une existence fulgurante. L'envie de vivre et de s'amuser s'estompe en faveur du souhait de survivre qui hante le monde entier à l'approche de la Seconde Guerre. L'architecture des cafés ne retrouvera plus jamais, durant le $\mathrm{xx}^{\mathrm{e}}$ siècle, l'enthousiasme de l'entre-deux-guerres.

Vue de l'esprit : du dessein au dessin

Le passage de l'étude historique à la conception architecturale se fait presque naturellement, puisque l'imagination a été sans cesse nourrie et stimulée par cette première phase, et que de nombreuses idées hantent l'esprit. Il s'agit maintenant de dépasser le " déjà vu ", d'éviter la paramnésie. Suivent des jours et des nuits d'angoisse pendant lesquels le cerveau travaille. Lorsque le projet est mûr le plus simple reste à faire : conclure, c'est-à-dire le dessiner.

39 Le choix du site a eu son importance. Comme tout étudiant architecte je suis libre de retenir, pour mon projet de diplôme, le programme, le site et les contraintes appropriés, tout comme j'ai choisi le sujet de mon mémoire. J'ai donc décidé de concevoir un café à la place Clichy (ill. 1). Une parcelle d'environ $130 \mathrm{~m}^{2}$ se trouve actuellement occupée uniquement en rez-de-chaussée par une partie du Café Wepler. Au-dessus, une énorme bâche publicitaire fait fonction de façade, dérobant ainsi au regard un vide dans la ville, vide difficile à combler en réalité pour des raisons liées à la réglementation urbaine.

La principale contrainte que je me suis imposée était d'utiliser les observations faites au cours de la recherche historique, de manière à intégrer l'histoire au projet. Un lecteur attentif pourra déceler la traduction en dessin de chaque remarque sur les cafés de l'entre-deux-guerres.

41 Une autre exigence, d'ordre urbanistique, était d'insérer la construction en posant le problème du renouvellement architectural, sans pour autant ignorer le cadre bâti existant. L'échelle de voisinage devait jouer son rôle, afin d'assurer une cohérence dynamique entre le projet et son environnement immédiat.

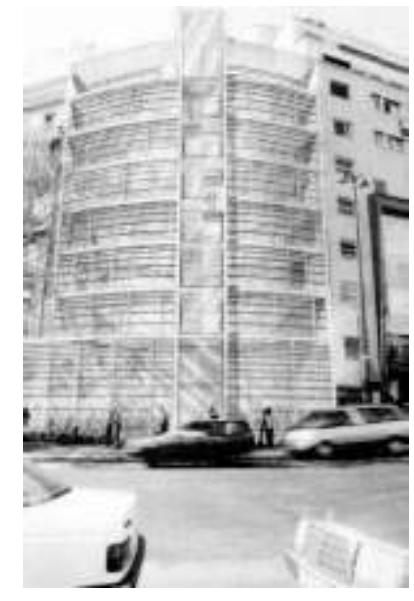

III. 1 - Vue du Café.

42 L'architecture n'est pas qu'un art, c'est aussi une science. À ce titre, j'ai associé à la conception les détails constructifs, soigneusement étudiés, afin de souligner leur rôle dans la création. La collaboration d'un ingénieur et d'un maître-verrier m'a assuré leur faisabilité. 

proposé un café à la verticale, afin d'obturer la « dent creuse » qui existe entre le cinéma Wepler et l'immeuble d'habitations voisin. Comme il a déjà été souligné, les cafés de l'entre-deux-guerres se développaient souvent sur plusieurs niveaux, l'idée n'est donc pas si absurde qu'elle paraît à première vue.

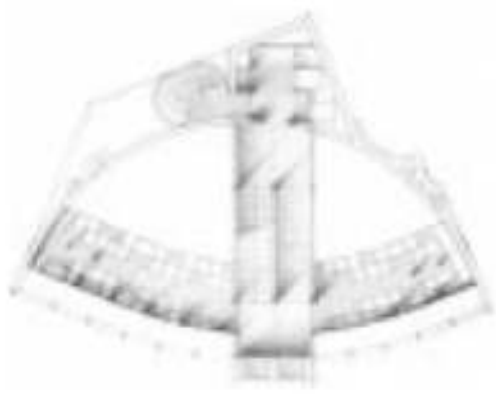

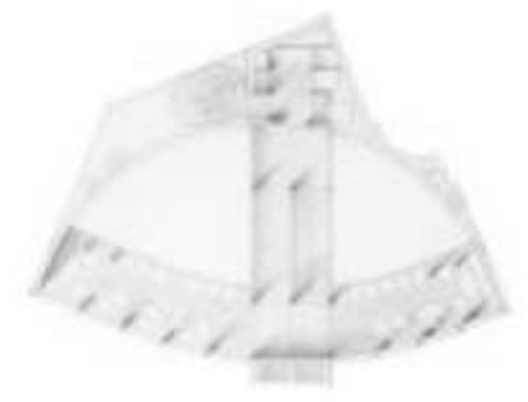

III. 2 - Plan des $1^{\text {er }}$ et $6^{\mathrm{e}}$ étages. Loges de théâtre et Salon.

$$
\begin{aligned}
& \text { que } \\
& \text { pour } \\
& \text { le } 4^{\mathrm{e}} \\
& \text { Le p }
\end{aligned}
$$
le $4^{\mathrm{e}}$, train pour le $5^{\mathrm{e}}$ et salon pour le $6^{\mathrm{e}}$ étage. , salon de lecture pour le $3^{\mathrm{e}}$, paquebot pour 

d'importance car, comme je l'ai constaté lors de la recherche, ils contribuent pleinement à créer du mouvement dans le café (ill. 3). Entièrement en verre, ils sont dématérialisés, de manière à amplifier l'espace et à mettre en exergue la présence humaine. Le même artifice, l'emploi du verre, fait disparaitre les tirants, principaux éléments de structure verticale qui soutiennent les niveaux ${ }^{24}$.

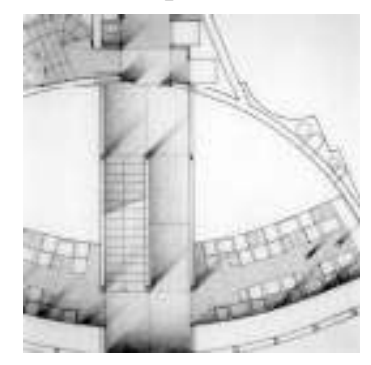

III. 3 - Détail de plan. 2e étage. Salle de jeux.

51 L'emploi des miroirs sur la paroi courbe du fond accentue l'impression d'agitation qui règne dans le café, tout en agrandissant l'espace. Le verre, traité de différentes manières, est omniprésent. Outre l'escalier central et les passerelles dont les marches et les planchers sont en verre translucide et les garde-corps en verre dépoli en dégradé, les rambardes de chaque niveau sont en verre feuilleté dépoli au marteau-piqueur. Ce traitement rappelle les bulles de gaz carbonique dans une flûte de champagne.

L'éclairage est intégré au plancher qui surplombe chaque niveau. Un complément d'éclairage par fibres optiques vient souligner la silhouette de l'escalier et des passerelles. Les mains courantes intègrent également un éclairage qui souligne leur mouvement courbe.

53 J'ai accordé une attention particulière au traitement de la façade sur la place Clichy. Le respect des alignements par rapport aux immeubles voisins a déterminé la hauteur de chaque niveau. Orientée plein sud, la façade nécessitait une occultation qui varie l'éclairement de l'espace intérieur et souligne la seule fonction désormais dévolue à une devanture - tout comme à la Maison du Café - celle de protection contre les intempéries. Elle est imaginée en verre extra-blanc, pour offrir un maximum de transparence. Chaque trame qui la compose est équipée d'un système de brise-soleil pouvant être actionné depuis les tables, ce qui permet aux clients d'adapter l'ensoleillement à leurs besoins ou de se créer une certaine intimité. Les lames métalliques, prises dans une double épaisseur de verre, pivotent sur leur propre axe central, en même temps qu'elles coulissent sur un rail vertical. La façade change ainsi à tout moment de la journée et de la nuit. Son dynamisme participe autant à l'atmosphère de la place qu'à l'ambiance du café.

Projet et théâtre. Prises de vues

54 Le voisinage immédiat a induit une grande hauteur pour le café. Le projet se développe donc sur dix niveaux, dont deux en sous-sol. Les étages ont une surface d'environ $30 \mathrm{~m}^{2}$ chacun. Le rez-de-chaussée s'étend également sous l'immeuble d'habitations (ill. 4) et comprend la salle de restaurant, la zone du bar, ainsi que les parties annexes : cuisines, office, bureau, etc. Afin que les clients du café puissent voir et être vus, les étages sont conçus sur le principe de deux théâtres à l'italienne qui s'imbriquent : le $1^{\text {er }}$ étage épouse la courbe de la paroi qui se trouve en fond de parcelle, tandis que le $2^{\mathrm{e}}$ suit la façade sur la place et ainsi de suite. Chaque étage est en retrait par rapport à l'étage situé en dessous.

Labyrinthe, 9 | 2001 
Cette organisation en gradins permet une meilleure vue sur l'ensemble de l'espace. La définition du café comme lieu de spectacle prend ainsi toute sa signification. Les niveaux disposés sur la façade principale permettent aux clients d'observer aussi le spectacle extérieur. Deux terrasses accompagnent l'espace proprement dit du café : une au rez-dechaussée, faisant la liaison entre la rue et l'intérieur, et une seconde au dernier niveau qui établit une relation entre l'espace céleste et l'espace du café. Cette relation est renforcée par la création d'un toit ouvrant pour le café (ill. 5). Par beau temps, les clients se trouvant à l'intérieur peuvent apercevoir le ciel.

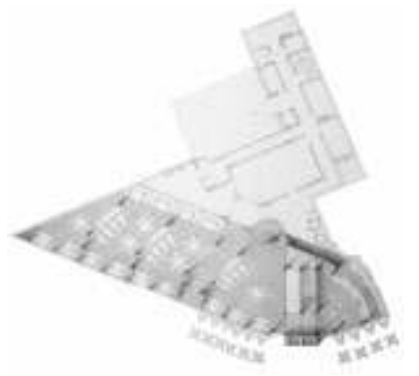

III. 4 - Plan du rez-de-chaussée.

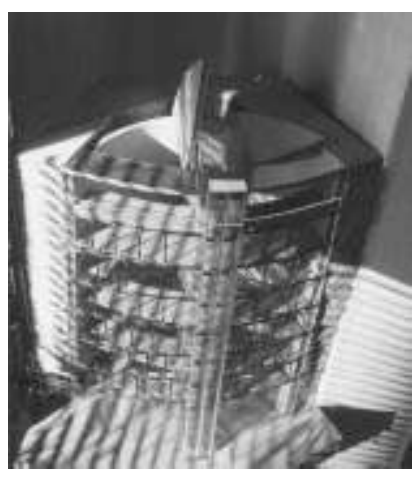

III. 5 - Vue du toit ouvrant.

Dessin et écriture. Parcours

L'écriture, tout comme le dessin, sont des cheminements vers l'insaisissable. On projette et on se projette. C'est par assimilation et articulation qu'un nouveau réel apparaît. Il devient donc normal de produire du sens en créant une relation entre littérature et architecture. Cela enrichit le projet et offre des solutions inattendues. Ainsi, par exemple, le rythme des barres métalliques qui constituent la " cage " de l'escalier central a été suggéré par le rythme des chapitres d'une fiction, Les Villes invisibles d'Italo Calvino (ill. 6).

L'impression de vertige qui domine l'espace s'inspire de l'ivresse provoquée par la lecture des textes d'Henri Michaux, comme : 


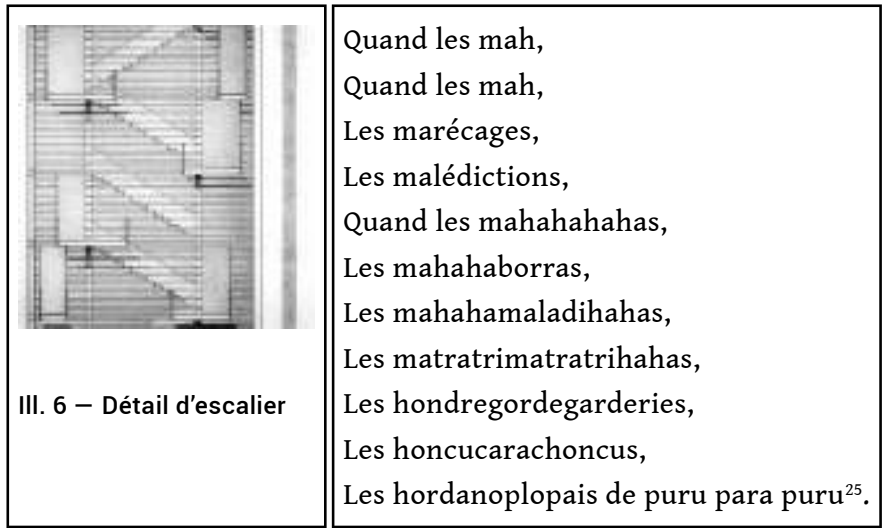

Café et enseigne. $\mathrm{Vu}$ !

Les remarques que j'ai pu faire lors de la recherche historique m'ont permis de comprendre l'apport des nouveaux caractères typographiques à la fabrication des enseignes de cafés. Celles-ci font partie des composantes de la façade et assurent un maximum de rendement publicitaire. Il était donc important que le projet proposé s'accompagne d'une recherche sur les caractères qui devaient composer l'enseigne. Les efforts que j'avais faits au début de la conception n'ont pas donné de résultats. Mais, lorsque l'espace fut dessiné, il m'est apparu possible d'écrire le mot CAFÉ, en utilisant le contour de certains étages. De nouveaux caractères étaient nés. La particularité de cette enseigne - fruit du hasard - est que la lettre $C$, troisième lettre de l'alphabet, correspond au plan du $3^{\mathrm{e}}$ étage, la lettre A, première lettre de l'alphabet correspond au plan du $1^{\mathrm{er}}$ étage, etc. (ill. 7).

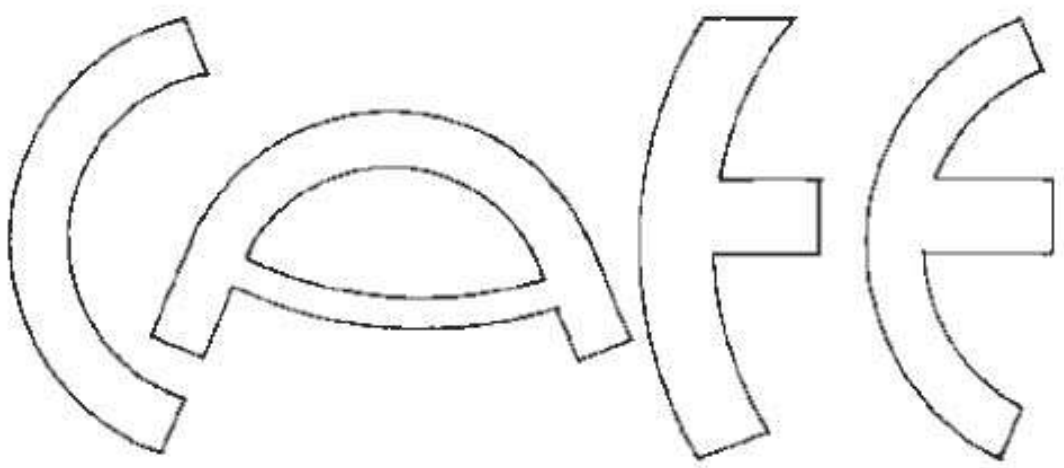

\section{7 - L'enseigne.}

Dessin à vue

La terrasse du dernier niveau est couverte, afin de permettre son utilisation par tous les temps. L'éclairage situé au plafond, obtenu à l'aide de fibres optiques, reprend les principales constellations visibles dans le ciel.

Pour la terrasse du rez-de-chaussée, le système de couverture suggère le principe des dessous-de-plat métalliques extensibles. Il utilise les mêmes lames de brise-soleil que pour la façade. Ceci permet, soit de régler le passage de la lumière, selon l'inclinaison des 
lames, soit d'avoir une obturation totale, lorsque les lames sont en position horizontale, soit de tout replier contre la façade, lorsque le dispositif n'est pas utilisé.

L'étude de l'architecture permet de saisir les interférences entre différentes histoires l'histoire de l'architecture, l'histoire sociale, l'histoire culturelle ou l'histoire des subjectivités - et de surprendre la manière dont celles-ci s'éclairent réciproquement. À ce titre, l'architecture peut participer au renouvellement des problématiques en histoire.

61 En associant simultanément les faits induits par l'histoire, les signes contenus par les textes, l'information fournie par l'iconographie et l'observation du quotidien, il a été possible de superposer l'« écriture » de l'architecture à celle de la vie. C'est par l'histoire que le jeu de l'invention et de la tradition, du détail et du global, du hasard et de la règle a été appréhendé, ce qui a rendu plus lucide l'acte de concevoir.

L'écriture de l'histoire a assouvi la volonté de savoir, tandis que la conception du projet a comblé le désir de création, qui anime généralement l'esprit de l'architecte.

\section{BIBLIOGRAPHIE}

ANDRIEU Pierre, Histoire du restaurant en France, s.l., éd. Journée vinicole, 1955, 230 p.

FARGUE Léon-Paul, Poisons, gravures de E.M. Burgin, Paris, Daragnes, 1946, 111 p.

FosCA François, Paris mon village... Histoire des cafés de Paris, Paris, Firmin-Didot et C $C^{\mathrm{ie}}, 1935,218$ p., 12 ill.

GRAND-CARTERET John, Raphaël et Gambrinus ou l'Art dans la brasserie, Paris, L. Westhausser, 1886, XXII-326 p.

LEMOINE Bertrand, RIVOIRARD Philippe, Paris, l'architecture des années trente, Paris, DAAVP, Lyon, Manufacture, 1987, $251 \mathrm{p}$.

VERNES Michel, «Cafés de Paris. À l'avenant de la ville », Architecture intérieure créée, octobrenovembre 1987, p. 89-95.

\section{NOTES}

Wissant Georges, Le Paris d'autrefois. Cafés et cabarets, Paris, J. Tallandier, 1928, 247 p.

* Cet article est la synthèse du diplôme soutenu en juin 1995 à l'École d'Architecture de Paris-La Villette (mémoire consultable à la bibliothèque de l'École). Jury : Michel Vernes, Philippe Boudon, Marc Mimram, Bernard Pictet.

1. Pour des raisons liées aux droits d'auteur ces documents ne peuvent être reproduits.

2. Léon Paul Fargue, Poisons, Paris, 1946, p. 85.

3. Taverne, caveau, cabaret, buvette, guinguette, estaminet, gargote, bistrot, café, etc.

4. Pour faciliter la lecture, c'est ce terme qui sera le plus souvent employé. 
5. Robert Mallet-Stevens, «Les Restaurants », L'Architecture d'Aujourd'hui n 12, décembre 1938, p. 45. Mallet-Stevens est un des rares protagonistes du Style International en France.

6. Robert J.Courtine, Un gourmand à Paris, Paris, B. Grasset, 1959, p. 23.

7. François Fosca, Histoire des cafés de Paris, Paris, Firmin-Didot, 1934, p. 213.

8. Léon-Paul Fargue, D’après Paris et Le piéton de Paris, Paris, Librairies de France, 1961, p. 135.

9. Id., op. cit., p. 310.

10. Robert-Robert, Le Guide du gourmand à Paris, Paris, B. Grasset, 1925, p. 97.

11. Comme La Maison Dorée (20, boulevard des Italiens), Le Café Riche (16, boulevard des Italiens), ou Le Café de Paris (angle du Boulevard des Italiens et de la rue Taitbout, puis 41, avenue de l'Opéra).

12. Jean Fayard, Dans le monde où l'on s'abuse, Paris, A. Fayard et Cie, 1925, p. 13-14.

13. Robert Sabatier, Boulevard, Paris, A. Michel, 1956, p. 251.

14. Odette Pannetier, Plaisirs forcés à perpétuité, Paris, éd. Prométhée, 1929, p. 20.

15. René Chavance, « Le bar "Presto" ou la décoration sans décor, Otto Bauer, architecte ", Mobilier et Décoration, août 1930, p. 49.

16. Marcel Zahar, Charles Siclis, Paris, L'Architecture d'Aujourd'hui, 1937, p. 6.

17. A.L. Solvet et Paul Solvet, Cafés, Brasseries, Restaurants, Strasbourg, Éd. d'architecture, d'industrie et d'économie rurale, s.d. [1932], p. 5.

18. Henri Hérault, « Les grands architectes - Siclis », Art et Médecine, n², novembre 1930, p. 33.

19. René Chavance, "Un café-restaurant de luxe "Le Colisée" ", Mobilier et Décoration, 1932, p. 371-378.

20. G.Brunon-Guardia, « La nouvelle Maison du Café, Place de l'Opéra », Art et Industrie, février 1934, p. 23.

21. «Café Boulevard Barbès à Paris ", L'Architecte, 1935, p. 58.

22. Léon-Paul Fargue, D'après Paris et Le Piéton de Paris, Paris, Librairies de France, 1961, p. 116-117. Le Piéton de Paris, dont est tiré l'extrait, semble avoir été publié pour la première fois en 1939.

23. Par exemple, Antony Goissaud, auteur d'un article sur le café-restaurant Le Berry (92, avenue des Champs-Élysées, Louis Grossard, architecte), paru dans La Construction moderne , 6 octobre 1929, p. 3, s'attarde sur la partie affectée en sous-sol aux téléphones et aux sanitaires. Il décrit les comptoirs des préposées, les vitrines qui ornent le hall et les panneaux de glace gravée qui décorent les portes des cabines téléphoniques et des toilettes.

24. Autre exemple d'interprétation des remarques faites au cours de la recherche historique : tandis que durant les années étudiées la structure du café est cachée par des habillages qui tendent à la faire disparaître, ici, la structure même est en verre, ce qui la rend presque invisible.

25. Henri Michaux, «L'Avenir », La Nuit remue, Paris, Gallimard, 1993, p. 189. 


\section{RÉSUMÉS}

L'exemple du café parisien permet de comprendre la superposition de l'écriture architecturale et de la vie sociale. Son cursus à l'École d'Architecture de Paris-La Villette a été pour l'auteur l'occasion d'étudier les cafés et les restaurants parisiens de l'entre-deux-guerres par le biais de son mémoire, Regards, réalisé en 1995. Et le projet qui accompagnait ce travail lui a permis de souligner les rapports qui peuvent s'établir entre la conception architecturale et l'histoire ou la littérature. Par l'intermédiaire de l'écriture elle a pu articuler des données flottantes, des mots et des composantes architecturales sans liaisons apparentes entre eux. Le projet présenté - un café, place Clichy - est une interprétation possible des observations qui découlent de la recherche historique. La démarche employée a permis des rapprochements interdisciplinaires inhabituels, ainsi qu'un renouvellement de la manière de concevoir, qui s'inspire du passé pour aboutir à une architecture innovante et hardie.

Crossing of genres, crossing of forms. The Parisian café Exchange of views - The example of the Parisian café allows us to understand the superposition of architectural writing and social life. Her programme in the Architectural School of Paris-La Villette has offered the author the opportunity to study cafés and Parisian restaurants between the wars, through her thesis, Looks, achieved in 1995. The project which was part of this work has allowed her to underline the links between the architectural conception and history or literature. By writing, she has articulated indefinite datas, words and architectural components which had no visible links between themselves. The project produced - a café, place de clichy in Paris - is a possible interpretation of the observations proceeding from historical research. The thought process used has permitted unusual interdisciplinary comparings and a renewal of the way to conceive, which is inspired by the past to result in an innovative and bold architecture.

\section{AUTEUR}

\section{JOANNE VAJDA}

Architecte diplômée de l'École d'Architecture de Paris-La Villette, elle prépare à l'EHESS une thèse en Histoire et Civilisations sur Le Rôle des voyages dans la transformation de l'espace parisien. 1855-1937, sous la direction de Christophe Prochasson. Elle a travaillé dans des agences d'architecture et de scénographie à Bucarest et à Paris. Elle participe depuis 1995 à un cours d'Histoire de la Ville à l'École d'Architecture de Paris-La Villette (en collaboration avec Michel Vernes). Une grande attention y est accordée au texte littéraire et à l'écriture comme moyens de production de l'espace. Cet enseignement tente de faire sortir le projet architectural de son isolement. 